incapable of further development. In anticipation of this exhaustion, in 1880 , a scheme had been prepared for tapping the Watts River, the average daily flow of which was estimated at 42 million gallons. The execution of the project was, however, delayed, and it was not until $189 \mathrm{I}$ that water from this source was actually turned on, when the name of the system, as well as of the river itself, was changed into Maroondah. The aqueduct is $4 \mathrm{I}$ miles long, with $25 \frac{1}{2}$ miles of open channel and twelve tunnels (three over a mile in length). The total cost of the Maroondah system amounted to $778,944 l$.

By 1907 the population had increased to 536,540 , and still further sources of supply were found necessary. In 1910, powers were granted to incorporate the O'Shannassy and Upper Yarra watersheds, and by I9I4 a supply of 20 million gallons per day was being obtained from the former river by means of an aqueduct $48 \frac{3}{4}$ miles in length. The Upper Yarra supplies remain to be exploited at some future date. The amount spent so far on the O'Shannassy scheme has been $426,890 l$.

\section{THE MECHANISM OF CHEMICAL CHANGE IN LIVING ORGANISMS.'}

I $F$ we take a general view over the large field of chemical reactions known, we notice that there is a great variety in the rate at which these reactions take place. Some, and especially those in which electrical forces play a part, reactions between inorganic ions, are practically instantaneous. They are familiar to all in the precipitations of the analytical chemist. Others, such as the hydrolysis of cane-sugar by water, are so slow as to be incapable of detection at ordinary temperatures, unless a very long time is allowed. There are, moreover, all possible stages intermediate between these extremes. Reactions between carbon compounds are, generally speaking, comparatively slow; but, as the name "organic" indicates; they are the characteristic chemical changes of the living cell.

Early workers in the domain of physiological chemistry-Schönbein, for example-were struck by the fact that reactions which require, in the laboratory, powerful reagents, such as strong acids and high temperatures, to make them take place at a reasonable rate, occur rapidly in the living organism at moderate temperatures and in the presence of extremely weak acids or alkalis. I may refer to the decomposition of proteins into their constituent amino-acids, which is a part of the normal process of digestion, but, when ordinary laboratory methods are used, requires boiling for several hours with concentrated hydrochloric or sulphuric acid.

The problem before us, then, is to discover how a slow reaction can be made to go faster. The most obvious and well-known method of doing this is by raising the temperature; but this is clearly out of the question in living cells. Another possibility is to make use of mass action, increasing by some means the effective concentration of the reacting substances; in this way the number of contacts per unit time would be raised. This is possible in the cell. There remains a third, the formation of an intermediate compound with another substance. This compound may be supposed to be both formed and again decomposed at a rapid rate, so that the total time taken is much less than that of the original reaction.

Now it is evident that something of the kind contemplated by these two latter possibilities is at the bottom of the process called "catalysis" by Berzelius. This chemist directed attention to the numerous cases

1 Abridged from a discourse delivered at the Royal Institution on March 24, by Prof. W. M. Payliss, F.R.S.

$$
\text { No. } 2434 \text {, VOL. } 97]
$$

known, even at his time, where the presence of a third substance brings about an enormous acceleration of a reaction, without itself taking part in it, so far as appears at first sight; at all events, this third substance reappears at the end unchanged. An example is the effect of finely divided platinum on hydrogen peroxide. Similar phenomena were known to Faraday, and described by him about the same time, but without giving them a special name.

Agents of this kind were soon discovered to be present in living cells. Such catalysts are called, for convenience, "enzymes," as suggested by Kühne, although there is no real scientific necessity for the name. That of "ferments" is still sometimes used, and is not now liable, as it was in Kühne's time, to cause confusion by application to living microbes.

Since catalysts are, as a rule, found unchanged at the end of their work, it is clear that they do not themselves afford energy for the purpose. Indeed, the energy change of a catalysed homogeneous system is the same as that of the reaction when proceeding at its ordinary slow rate. How, then, do they act?

The first thing to note with respect to enzymes is that they are capable of activity in media in which they are insoluble. Whatever may be the nature of this activity, therefore, it is exerted by the surface of the catalyst. We may then reasonably ask, as the most obvious hypothesis, is there ground for holding that the increased rate of reactions brought about by enzymes is effected by increase of concentration of the reagents at the surface and consequent acceleration of the reaction by mass action? We know that substances which lower surface energy of any form are concentrated at such boundary surfaces. The process is well known as "adsorption," and is a consequence of the operation of the principle of Carnot and Clausius, which states that decrease of free energy always occurs, if it is possible for it to do so. In fact, such an explanation was given by Faraday of the effect of metallic platinum in causing combination of oxygen and hydrogen gases. Although the name "adsorption" was not used in this description, Faraday had very clear ideas of the process, and gives several interesting cases. He showed that the necessary condition for the activity of platinum in the case referred to is a chemically clean surface, in order that the gases may condense on it. It matters not whether the removal of deposit is effected by mechanical polishing ; by the action of acid or of alkali ; by oxidation or reduction-making it either anode or kathode in an electrolytic cell will serve. It should be mentioned that this view did not receive universal acceptance, but the fact that it recommended itself to the keen insight of Faraday is powerful evidence in its favour.

I would not venture to state that this hypothesis is yet in a position to explain all the facts met with in the action of enzymes themselves, but it is remarkable how many receive a satisfactory account. We are at once confronted by the difficulty of the considerable number of different enzymes. But we must not forget that adsorption is controlled by a great number of factors in addition to mechanical surface tension. All those properties which suffer modification at phase boundaries play their part-electrical charge, solubility, compressibility, even chemical reaction itself, may be mentioned. Moreover, as Hardy has pointed out the act of condensation in itself may well be accompanied by the manifestation of molecular forces which result in increased chemical potential of the reacting substances. It is clear that experimental decision of the questions involved is almost impossible until we have in our hands pure preparations of enzymes. We cannot as yet exclude the possibility of the formation of intermediate chemical compounds 
between enzyme and substrate, but their existence has not been demonstrated, and what I may venture to call Faraday's view has the advantage of simplicity, and thus the support of William of Occam's "razor."

The important question of the synthetic action of enzymes demands a little attention at this point. All reactions may be regarded as being, in principle, reversible or balanced, and the greater part of those of the living organism are found experimentally to be so. If we take for consideration those enzymes the action of which consists in the addition or removal of the elements of water, we find that, as would be expected from the law of mass action, the position of equilibrium in the presence of a large excess of water is very near to that of complete hydrolysis, and this is the state of affairs in the usual laboratory experiments. On the other hand, the less water is present, the greater is the preponderance of the oppositesynthetic-aspect. Take the classical case of ethyl acetate. If the ester and water are mixed in molecular proportions, hydrolysis to acid and alcohol occurs until two-thirds of the ester are decomposed. Moreover, the same final composition is obtained if we commence with acid and alcohol, and so work in the other direction. But these reactions proceed by themselves with extreme slowness, taking months before coming to an end. But the presence of a catalyst, such as mineral acid, brings about equilibrium in an hour or so, and we notice that it is the same as the spontaneous one. An enzyme, known as lipase, also brings about equilibrium rapidly. The important point in respect of the mechanism of living cells is that by changing the available amount of water, the reaction may be made to proceed in either direction at will. The series of curves given by Armstrong and Gosney (Proc. Roy Soc., 88 B, p. I76) show this fact very clearly. Further, if the equilibrium is brought about rapidly, even if to any position except that of complete change in one or the other direction, the enzyme must accelerate both reactions, and any hypothesis of special "synthesising" enzymes is superfluous. This is essentially the position taken by van't Hoff in the work with which he was engaged at the time of his death. What is required, then, is a means by which the cell is enabled to change the available water at the disposal of reactions occurring therein. We do not as yet know the precise nature of such mechanisms, but there is reason to believe that they are provided by changes in the surface area of colloidal constituents or in the power of imbibition possessed by certain contents of the cell.

We here come across an interesting probletn which cannot be said to be solved satisfactorily at present. We have seen that the equilibrium position of an ester system when reached rapidly under the action of a soluble catalyst is the same as the spontaneous one. But there is a certain difference when a heterogeneous catalyst, or enzyme, is used. Nevertheless, the equilibrium is a true one, being in the same position when approached from either end. The amount of butyric acid combined as amyl ester in a particular system under acid catalysis was found by Dietz to be 88 per cent. of the total; under the action of the enzyme lipase it was only 75 per cent. This fact has given rise to various suggestions, and has troubled people's minds because it appears to give a possibility of evading the second law of energetics. Now, it was pointed out to me by Prof. Hopkins that, on the hypothesis of a rapid attainment of equilibrium by condensation on the surface of the enzyme, it is necessary, if the natural equilibrium is to be unaltered, that adsorption of all the components of the system should be the same proportion of each, because the position of equilibrium must be the same on the surface of the enzyme as that which results in the body of the solu- tion. In the presence of a large excess of water, it does not seem likely that a difference of equilibrium owing to this cause could be detected. But this should be possible when the equilibrium position is nearer the middle, so to speak, and $I$ am at present engaged in experiments on the question. At any rate, difference in adsorption may be the cause of the phenomenon of Dietz. It would simply imply that water is adsorbed by the enzyme in relatively larger proportion than the other constituents of the system. It should be remembered that the solvent in these experiments was amyl alcohol containing about 8 per cent. of water, and, as Arrhenius has shown, all substances present are adsorbed, although the laws governing the relative proportion of these various substances are not yet completely worked out.

We see, by consideration of the facts relating to the action of enzymes, how important a part is played by changes in the rate of reactions, and there are two. further points to which attention has been directed by Prof. Hopkins. Take, first, a series of reversible reactions in which the products of one form the starting point of the next following :-

$$
\mathrm{A} \rightleftharpoons \mathrm{B} \rightleftharpoons \mathrm{C} \rightleftharpoons \mathrm{D} \rightleftharpoons \text { etc. }
$$

If the rate at which $B$ is converted into $C$ is greater than that at which A changes into B, it is obvious that the amount of $B$ present at any moment may be extremely small, although the whole of the final products have passed through the stage. The fact warns us from estimating the importance of any particular constituent of the cell by the quantity to be obtained.

The second point is this. Suppose that there are two independent reversible reactions, both leading to the same product, $\mathbf{C}$.

$$
\mathrm{B} C
$$

and that $\mathrm{A} \longrightarrow \mathrm{C}$ is more rapid or easier than $\mathrm{B} \longrightarrow \mathrm{C}$. This latter reaction will be practically absent, being balanced by the excess of $\mathrm{C}$. But, if the former reaction is abolished by removal of $A$, then $B \rightarrow C$ will take place in proportion as $C$ is used up in other reactions. Thus, under special conditions, a reaction may take place which is not detectable under normal conditions, although capable of taking place.

One of the most difficult questions is the manner in which the various components of the cell are prevented from entering into chemical reaction except when required. Enzymes, for example, are not always in activity. The conception which states that the cell consists of numerous minute "reaction chambers," separated from one another by membranes, seems to present most possibilities. These membranes must be regarded as capable of removal and of reconstruction, or reversible as regards their permeability. The food vacuoles of an Amœba may serve as an illustration of such chambers on a comparatively large scale. $I_{n}$ these vacuoles digestion processes are going on independently of other reactions in various parts of the same cell protoplasm, although this latter behaves as a liquid.

The general conclusion to which we arrive is that velocity of reaction plays an exceedingly important part in the regulation of cell mechanics. I venture to think that the conception is destined to replace static points of view, such as that of "lock and key" or the fitting together of molecular groupings. That there is still very much to be discovered is obvious. We have to find out how the living cell is able to modify and adjust together the large number of reactions known to the chemist. The study of the methods by which the rate of these reactions is affected is one of the most valuable of those accessible to us. 